\title{
Batten disease: biochemical and molecular characterization revealing novel PPT1 and TPP1 gene mutations in Indian patients
}

\author{
Jayesh Sheth ${ }^{1 *}$, Mehul Mistri ${ }^{1}$, Riddhi Bhavsar ${ }^{1}$, Dhairya Pancholi ${ }^{1}$, Mahesh Kamate ${ }^{2}$, Neerja Gupta ${ }^{3}$,
} Madhulika Kabra ${ }^{3}$, Sanjiv Mehta ${ }^{4}$, Sheela Nampoothiri ${ }^{5}$, Arpita Thakker ${ }^{6}$, Vivek Jain ${ }^{7}$, Raju Shah ${ }^{8}$ and Frenny Sheth ${ }^{1}$

\begin{abstract}
Background: Neuronal ceroid lipofuscinoses type I and type II (NCL1 and NCL2) also known as Batten disease are the commonly observed neurodegenerative lysosomal storage disorder caused by mutations in the PPT1 and TPP1 genes respectively. Till date, nearly 76 mutations in PPT1 and approximately 140 mutations, including large deletion/duplications, in TPP1 genes have been reported in the literature. The present study includes 34 unrelated Indian patients (12 females and 22 males) having epilepsy, visual impairment, cerebral atrophy, and cerebellar atrophy.

Methods: The biochemical investigation involved measuring the palmitoyl protein thioesterase 1 and tripeptidy peptidase I enzyme activity from the leukocytes. Based on the biochemical analysis all patients were screened for variations in either PPT1 gene or TPP1 gene using bidirectional Sanger sequencing. In cases where Sanger sequencing results was uninformative Multiplex Ligation-dependent Probe Amplification technique was employed. The online tools performed the protein homology modeling and orthologous conservation of the novel variants.

Results: Out of 34 patients analyzed, the biochemical assay confirmed 12 patients with NCL1 and 22 patients with NCL2. Molecular analysis of PPT1 gene in NCL1 patients revealed three known mutations (p.Val181Met, p.Asn110Ser, and p.Trp186Ter) and four novel variants (p.Glu178Asnfs*13, p.Pro238Leu, p.Cys45Arg, and p.Val236Gly). In the case of NCL2 patients, the TPP1 gene analysis identified seven known mutations and eight novel variants. Overall these 15 variants comprised seven missense variants (p.Met345Leu, p.Arg339Trp, p.Arg339GIn, p.Arg206Cys, p.Asn286Ser, p.Arg152Ser, p.Tyr459Ser), four frameshift variants (p.Ser62Argfs*19, p.Ser153Profs*19, p.Phe230Serfs*28, p. Ile484Aspfs*7), three nonsense variants (p.Phe516*, p.Arg208*, p.Tyr157*) and one intronic variant (g.2023_2024insT). No large deletion/duplication was identified in three NCL1 patients where Sanger sequencing study was normal.

Conclusion: The given study reports 34 patients with Batten disease. In addition, the study contributes four novel variants to the spectrum of PPT1 gene mutations and eight novel variants to the TPP1 gene mutation data. The novel pathogenic variant p.Pro238Leu occurred most commonly in the NCL1 cohort while the occurrence of a known pathogenic mutation p.Arg206Cys dominated in the NCL2 cohort. This study provides an insight into the molecular pathology of NCL1 and NCL2 disease for Indian origin patients.
\end{abstract}

Keywords: PPT1, TPP1, Batten disease, Neuronal ceroid lipofuscinoses (NCL)

\footnotetext{
* Correspondence: jshethad1@gmail.com

${ }^{1}$ FRIGE's Institute of Human Genetics, FRIGE House, Jodhpur Gam Road,

Satellite, Ahmedabad, Gujarat 380015, India

Full list of author information is available at the end of the article
}

(c) The Author(s). 2018 Open Access This article is distributed under the terms of the Creative Commons Attribution 4.0 International License (http://creativecommons.org/licenses/by/4.0/), which permits unrestricted use, distribution, and reproduction in any medium, provided you give appropriate credit to the original author(s) and the source, provide a link to the Creative Commons license, and indicate if changes were made. The Creative Commons Public Domain Dedication waiver (http://creativecommons.org/publicdomain/zero/1.0/) applies to the data made available in this article, unless otherwise stated. 


\section{Background}

The neuronal ceroid lipofuscinoses (NCLs) are a group of inherited lysosomal storage disorder causing severe neurodegeneration due to neuronal loss (brain and retina), and accumulation of lipopigments in many cell types, including neurons. The NCLs incidence rate worldwide is 1 to 8 in 100,000 live births [1].

The NCLs share common clinical presentations like epilepsy, loss of motor and cognitive function, visual impairment, and premature death [2]. Though usually observed in childhood, the age of onset of the disease varies. Considering this, NCLs were initially classified into four groups - infantile (INCL-Haltia-Santavuori disease), late infantile (LINCL-Jansky-Bielschowsky disease), juvenile (Batten-Spielmeyer-Vogt disease) and adult (Kufs disease) [3]. Eventually, allelic heterogeneity in NCLs was identified due to an advancement in biochemical and genetic techniques and hence a new approach of molecular classification and diagnostic algorithms was designed [4, 5].

Until now, 14 types of NCL are identified (NCL1-NCL14) however the most commonly observed form are NCL1, NCL2, and NCL3 [6]. These NCLs subtypes are autosomal recessively inherited except for NCL4B, inherited in an autosomal dominant form [7]. An exception to the above inheritance pattern is a uniparental disomy case in NCL which occurred due to complete isodisomy of chromosome 8 , leading to homozygosity of a maternally-inherited deletion in NCL8 [8]. Until May 2015, total 515 changes in 13 human genes have been reported in NCLs [9].

NCL1 (OMIM\#256730) representing the early infantile disease, results due to a mutation in PPT1 (palmitoyl-protein thioesterase 1 ; OMIM*600722) gene located at 1p34.2. The gene codes for a lysosomal enzyme called palmitoyl-protein thioesterase 1 (PPT1) whose function is to remove fatty acids attached in thioester linkages to cysteine residues in the protein. The downstream effect of PPT1 deficiency involves deregulated cellular processes like vesicular trafficking, synaptic function, lipid metabolism, neural specification, and axon connectivity [10]. In addition, a study by Lyly et al. established that an alteration in cholesterol metabolism and ectopic F1-ATP synthase resulted due to PPT1 deficiency [11]. According to the NCL mutation and patients' database 76 changes have been reported in PPT1 gene [9]. Amongst these, the mutation p.Arg122Trp has a founder effect in Finnish population with NCL1 [12]. This mutation causes a defect in the transport of the PPT1 from the endoplasmic reticulum to lysosomes [12, 13]. The mutation p.Thr75Pro and p.Leu10Ter have a founder effect in Scotland [14].

NCL2 (OMIM\#204500), representing the late infantile disease, results due to a defect in TPP1 (tripeptidyl peptidase I; OMIM*607998) gene at the locus 11p15.4. This gene encodes the instruction for making the lysosomal enzyme called tripeptidyl peptidase 1 (TPP1). TPP1 deficiency results in accumulation of ceroid lipofuscin, an autofluorescent storage material, in cell's lysosomes. Total 140 disease-causing mutations are in the TPP1 gene of the patients with NCL2 [9]. The most common TPP1 gene mutations are c.509-1G > C and p.Arg208Ter [14]. The mutation p.Gly284Val seems to be predominant in Canada suggesting a possible founder effect [14].

The genetics of NCL1 and NCL2 remain unknown in India. Hence, the aim of the present study is to identify the molecular spectrum and common molecular marker of these diseases in Indian patients. The study also aims to support the correlation between the null or reduced enzyme activity and the mutations causing disease and clinical phenotype in NCL1 and NCL2 patients.

\section{Methods}

\section{Patients}

The patients in the present study were the clinical cases referred by pediatric neurologist and pediatricians from collaborating centres. The study is in accordance with the tenets of the Helsinki Declaration. The Ethics committee of the Foundation for Research in Genetics and Endocrinology (FRIGE) at the Institute of Human Genetics approved the study. As per the institutional ethics committee guidelines, a written informed consent for investigation and publication of the data was obtained from the parents/guardian of the patients. The 34 unrelated patients ( 22 males and 12 females) presented common clinical indications like epilepsy, cerebral atrophy, and cerebellar atrophy. They were in the age range of 4 months to 9 years at the time of investigations and were referred from different geographical/ethnic background in the time from 2015 to 2017 with a clinical suspicion of Batten disease (12 patients with NCL1, and 22 patients with NCL2). Total 16 patients (47.05\%) had parental consanguinity. Table 1 provides the clinical details and the demographic profile of the patients.

\section{Biochemical investigations}

Leukocyte and genomic DNA (gDNA) isolation was carried out from six milliliters of blood, drawn from each patient in ethylenediaminetetraacetic acid (EDTA) vacutainer.

\section{Palmitoyl protein thioesterase 1 (PPT1) enzyme activity} The patients suspected with the NCL1 disease were investigated for the lysosomal enzyme Palmitoyl protein thioesterase 1 (PPT1; EC 3.1.2.22) activity using a previously described protocol [15]. In brief, the samples were incubated with tritinX 100, $\beta$-glucosidase, dithiothreitol and the substrate 4-Methylumbelliferyl-6-sulpho-palmitoyl- $\beta$-D-glucoside at $37^{\circ} \mathrm{C}$ for $1 \mathrm{~h}$. The fluorescence was measured at $4 \mathrm{mu}$. 
Table 1 Clinical details and demographic profile of the patients with Batten disease (NCL1 and NCL2)

\begin{tabular}{|c|c|c|c|}
\hline & $\begin{array}{l}\text { Total patients } \\
n=34\end{array}$ & $\begin{array}{l}\text { NCL1 } \\
n=12\end{array}$ & $\begin{array}{l}\text { NCL2 } \\
n=22 \\
\end{array}$ \\
\hline Age at the time of investigation (years) & $4.46 \pm 2.30$ & $3.03 \pm 1.80$ & $5.25 \pm 1.93$ \\
\hline \multicolumn{4}{|l|}{ Gender } \\
\hline Male & $22(64.7 \%)$ & $8(66.6 \%)$ & $14(63.6 \%)$ \\
\hline Female & $12(35.3 \%)$ & $4(33.4 \%)$ & $8(36.4 \%)$ \\
\hline \multicolumn{4}{|l|}{ Regional distribution } \\
\hline East India & $2(5.9 \%)$ & 0 & $2(9.09 \%)$ \\
\hline West India & $9(26.4 \%)$ & $2(16.7 \%)$ & $7(31.81 \%)$ \\
\hline North India & $3(8.8 \%)$ & $1(8.3 \%)$ & $2(9.09 \%)$ \\
\hline South India & $20(58.9 \%)$ & $9(75 \%)$ & $11(50 \%)$ \\
\hline \multicolumn{4}{|l|}{ Clinical presentation } \\
\hline Epilepsy & $31(91.2 \%)$ & $10(83.33 \%)$ & $21(95.45 \%)$ \\
\hline Visual impairment & 19 (55.9\%) & $3(25 \%)$ & $16(72.72 \%)$ \\
\hline Cerebral atrophy & $22(64.7 \%)$ & $6(50 \%)$ & $16(72.72 \%)$ \\
\hline Cerebellar atrophy & $27(79.41 \%)$ & $6(50 \%)$ & $21(95.45 \%)$ \\
\hline
\end{tabular}

Tripeptidy peptidase I (TPP1) enzyme activity

The NCL2 suspected patients were investigated for the Tripeptidy peptidase 1 (TPP1; EC 3.4.14.9) lysosomal enzyme activity using a previously described protocol [16]. In brief, the samples were mixed with EDTA/acetate/TritonX 100 in chilled condition. The substrate Ala-Ala-Phe-7-amido-4-methylcoumarin was added on limited intervals and incubated at $37^{\circ} \mathrm{C}$ for $1 \mathrm{~h}$. Chloroacetate/acetate was added following the incubation and the fluorescence was measured at $460 \mathrm{~nm}$.

\section{Molecular investigation DNA extraction}

DNA isolation followed the standard salting-out method and was quantified using a QIAxpert (Cat. No: 9002340) from Qiagen [17]. Sample purification was performed using The Genomic DNA Clean \& Concentrator $^{\text {rm }}-25$ $\left(\mathrm{DCC}^{\mathrm{mm}}\right)$ Kit, from Zymo Research, Irvine, California, U.S.A (Cat. No. D4064) and were stored at $-20^{\circ} \mathrm{C}$ until investigation.

\section{Single gene sequencing (PPT1 and TPP1 gene)}

The PPT1 and TPP1 genes were amplified using primer sets listed in the Additional file 1. The protocol followed 37 cycles consisting of initial denaturation $\left(94^{\circ} \mathrm{C} ; 5 \mathrm{~min}\right)$, denaturation $\left(94{ }^{\circ} \mathrm{C}\right.$; $\left.30 \mathrm{~s}\right)$, annealing $\left(58^{\circ} \mathrm{C}-67^{\circ} \mathrm{C} ; 45 \mathrm{~s}\right)$, elongation $\left(72{ }^{\circ} \mathrm{C} ; 1 \mathrm{~min}\right)$, and final elongation $\left(72{ }^{\circ} \mathrm{C} ; 5\right.$ $\mathrm{min})$. The polymerase chain reaction (PCR) products were run on the $2.5 \%$ agarose gel and visualized under ultraviolet transilluminator.

The capillary electrophoresis technology driven fluorescent dye-labeled genetic analysis system performed the
Sanger sequencing on the Applied Biosystems ${ }^{\text {Tt }}$ SeqStudio $^{\text {th }}$ Genetic Analyzer with SeqStudio $^{\text {ma }}$ Data Collection Software using a previously described protocol [18].

\section{Multiplex ligation-dependent probe amplification (MLPA) analysis}

The procedure followed the manufacturer's recommendations of using gDNA (100 ng) and P470-A1 NCL probe mix (MRC-Holland, Amsterdam, the Netherlands). DNA was denatured $\left(98^{\circ} \mathrm{C} ; 5 \mathrm{~min}\right)$ and hybridized (overnight; $60^{\circ} \mathrm{C}$ ) with the SALSA probe mix P470. The samples were then Ligase $\left(54^{\circ} \mathrm{C} ; 15 \mathrm{~min}\right)$ and incubated at $98^{\circ} \mathrm{C}$ for 5 min to stop the reaction. The PCR amplification was carried out with the specific SALSA FAM PCR primers. Amplified products were run on the ABI 3130 Genetic Analyzer (Applied Biosystems, USA) and the MLPA peak patterns were analyzed in control and test samples to detect the copy number differences of the exons.

\section{In silico analysis \\ Prediction of the functional effect of the variants}

The variants identified were looked up in public databases like The Human Gene Mutation Database (http://www.hgmd.cf.ac.uk), SNP database (http:// www.ncbi.nlm.nih.gov/SNP/index.html) and UCL-Lon don's Global university database (http://www.ucl.ac.uk/ncl). The in silico tools like MutationTaster2, SIFT, FATHMM, PolyPhen2, PROVEAN, and MutationAssessor predicted the pathogenicity of the coding and non-coding DNA variants and amino acid substitution. 


\section{Homology modeling, structure validation and protein stability of the novel variants}

The wild-type template crystallographic structures of the PPT1 (PDB ID: 1EI9) and TPP1 (PDB ID: 1EDY) were used to calculate the Root Mean Square Deviation (RMSD) of the novel mutant protein structures [19].

\section{Orthologous conservation of the residues harboring the novel variant}

Clastal Omega (an online multiple sequence alignment program) aligned the PPT1 (NP_000301) and TPP1 (NP_000382) protein sequence of Homo sapiens with different species to check the conservation of the residues incorporating novel variants [19].

\section{Results}

In the present study the clinical assessment, biochemical and molecular investigation confirmed the diagnosis of 34 patients with Batten disease (12 with NCL1 and 22 with NCL2). The most common clinical indications observed in these patients were epilepsy (91.2\%). The neuroimaging study including Computed Tomography (CT scan) and/or brain Magnetic Resonance Imaging (MRI) from all the patients also revealed cerebral atrophy (64.7\%), and cerebellar atrophy (79.41\%).

\section{Biochemical investigations}

A significant deficiency of PPT1 and TPP1 enzyme activity was observed in the leukocytes of twelve patients with NCL1 and twenty-two patients with NCL2 respectively. The patients' enzyme activity reduced to 0 to $2.8 \%$ compared to the control value (Tables 2 and 3 ).

\section{Molecular analysis \\ Bi-directional sanger sequencing (PPT1 gene)}

The biochemical investigation confirmed 12 patients with NCL1. Sanger sequencing identified five homozygous missense variants, one frameshift variant, and one nonsense variant from total nine patients (Table 2).

\section{Known mutation detected in the PPT1 gene}

In total three known variants were revealed by Sanger sequencing. Patient $\mathrm{P}_{1}$ and $\mathrm{P}_{2}$ harbor the pathogenic homozygous mutations p.Val181Met in exon 6 and p.Asn110Ser in exon 3 respectively $[20,21]$. Patient $P_{3}$ carried compound heterozygous mutation p.Trp186Ter (known pathogenic) in exon 6 and a novel variant p.E178Nfs*13 in exon 5 [22].

\section{Novel variants detected in PPT1 gene}

Overall, four novel variants amongst nine patients responsible for NCL1 were detected (Fig. 1). Patient $\mathrm{P}_{4}$ to $\mathrm{P}_{7}$ were homozygous for the variant p.Pro238Leu in

Table 2 Biochemical and molecular alalysis of patients with NCL1 disease

\begin{tabular}{|c|c|c|c|c|c|c|c|}
\hline \multirow{2}{*}{$\begin{array}{l}\text { Patient } \\
\text { ID }\end{array}$} & \multirow{2}{*}{$\begin{array}{l}\text { Palmitoyl } \\
\text { protein } \\
\text { thioesterase } \\
\text { activity } \\
\text { (nmol/hr./ } \\
\text { mg protein) }\end{array}$} & \multicolumn{2}{|l|}{ Molecular analysis } & \multicolumn{2}{|l|}{ Allele Frequency } & \multirow{2}{*}{$\begin{array}{l}\text { dbSNP } \\
\text { reference } \\
\text { sequence }\end{array}$} & \multirow[t]{2}{*}{ Reference } \\
\hline & & Variant location (PPT1 gene ${ }^{c}$ ) & Zygosity & 1000 Genomes & ExAC & & \\
\hline$P_{1}$ & 0.0 & Ex6:c.541G>A/p.V181M & Hom & NR & 0.0001648 & rs148412181 & [19] \\
\hline$P_{2}$ & 3.6 & Ex3:c.329A>G/p.N110S & Hom & NR & 0.0001813 & rs142894102 & {$[20]$} \\
\hline \multirow[t]{2}{*}{$P_{3}$} & 3.6 & Ex6:c.558G>A/p.W186 & Com Hetz & NR & 0.000008237 & rs386833656 & {$[21]$} \\
\hline & & \multicolumn{2}{|l|}{ Ex5:c.532_532delG/p.E178Nfs*13 } & NR & NR & rs878853325 & In this study \\
\hline$P_{4}$ & 3.1 & Ex7:c.713C>T/p.P238L & Hom & NR & NR & rs878853322 & In this study \\
\hline$P_{5}{ }^{a}$ & 0.01 & & & & & & \\
\hline$P_{6}$ & 0.72 & & & & & & \\
\hline$P_{7}$ & 1.07 & & & & & & \\
\hline$P_{8}$ & 7.3 & Ex2:c.133T>C/p.C45R & Hom & NR & NR & rs878853323 & In this study \\
\hline$P_{9}$ & 0.36 & Ex7:c.707T>A/p.V236G & Hom & NR & NR & rs878853324 & In this study \\
\hline$P_{10}$ & 5.2 & Not found & - & - & - & - & - \\
\hline$P_{11}$ & 7.4 & & & & & & \\
\hline$P_{12}$ & 3.1 & & & & & & \\
\hline
\end{tabular}


Table 3 Biochemical and molecular analysis of patients with NCL2 disease

\begin{tabular}{|c|c|c|c|c|c|c|c|}
\hline \multirow{2}{*}{$\begin{array}{l}\text { Patient } \\
\text { ID }\end{array}$} & \multirow[b]{2}{*}{$\begin{array}{l}\text { Tripeptidyl } \\
\text { Peptidase-l } \\
\text { activity } \\
\text { (nmol/hr./ } \\
\text { mg } \\
\text { protein) }\end{array}$} & \multicolumn{2}{|l|}{ Molecular analysis } & \multicolumn{2}{|l|}{ Allele Frequency } & \multirow{2}{*}{$\begin{array}{l}\text { dbSNP } \\
\text { reference } \\
\text { sequence }\end{array}$} & \multirow[t]{2}{*}{ Reference } \\
\hline & & Variant location (TPP1 gene ${ }^{\complement}$ ) & Zygosity & 1000 Genomes & EXAC & & \\
\hline$P_{13}$ & 10.2 & Ex8:c.1033A>C/p.M345L & Hetz & 0.0030 & 0.001796 & rs141482368 & {$[22]$} \\
\hline$P_{14}$ & 4.5 & Ex8:c.1015C>T/p.R339W & Hom & NR & 0.00001648 & rs750428882 & [23] \\
\hline$P_{15}$ & 4.9 & Ex8:c.1016G>A/p.R339Q & Hom & NR & 0.000008241 & rs765380155 & [2] \\
\hline$P_{16}$ & 11.9 & Ex12:c.1546_1547delTT/p.F516* & Hom & NR & NR & - & [24] \\
\hline$P_{17}$ & 5.4 & Ex6:c.616C>T/p.R206C & Hom & NR & 0.00001649 & rs28940573 & {$[25]$} \\
\hline$P_{18}{ }^{*}$ & 8.2 & & & & & & \\
\hline$P_{19}$ & 4.7 & & & & & & \\
\hline$P_{20}$ & 2.9 & & & & & & \\
\hline$P_{21}{ }^{a}$ & 0.0 & & & & & & \\
\hline$P_{22}{ }^{a}$ & 9.7 & Ex6:c.622C>T/p.R208* & Hom & NR & 0.0002 & rs119455955 & {$[26]$} \\
\hline \multirow[t]{2}{*}{$P_{23}$} & 6.5 & Ex7:c.857A>G/p.N286S & Com Hetz & NR & NR & rs119455958 & {$[27]$} \\
\hline & & Ex3:c.184delT/p.S62Rfs*19 & & NR & NR & NR & In this study \\
\hline$P_{24}$ & 9.1 & Ex5:c.456G>C/p.R152S & Hom & NR & NR & rs 869025274 & In this study \\
\hline$P_{25}{ }^{a}$ & 0.0 & Ex11:c.1376A>C/p.Y459S & Hom & NR & NR & rs864309505 & In this study \\
\hline$P_{26}$ & 4.6 & & & & & & \\
\hline $\mathrm{P}_{27}^{\mathrm{a}}$ & 0.3 & Ex5:c.455_488del/p.S153Pfs*19 & Hom & NR & NR & NR & In this study \\
\hline $\mathrm{P}_{28}{ }^{\mathrm{a}}$ & 0.0 & Ex5:c.471C>A/p.Y157* & Hom & NR & NR & rs553522118 & In this study \\
\hline \multirow[t]{2}{*}{$P_{29}$} & 9.2 & Ex7:c.689_689delT/p.F230Sfs*28 & Com Hetz & NR & NR & NR & In this study \\
\hline & & Ex12:c.1449_1450insG/p.1484Dfs*7 & & NR & NR & NR & In this study \\
\hline$P_{30}{ }^{a}$ & 0.4 & In4:g.2023_2024insT & Hom & NR & NR & NR & In this study \\
\hline$P_{31}{ }^{a}$ & 0.0 & & & & & & \\
\hline$P_{32}$ & 3.5 & Not found & - & - & - & - & - \\
\hline$P_{33}$ & 5.6 & & & & & & \\
\hline$P_{34}$ & 8.2 & & & & & & \\
\hline
\end{tabular}

exon 7. Patient $\mathrm{P}_{8}$ and $\mathrm{P}_{9}$ presented homozygous missense variants p.Cys45Arg in exon 2 and p.V236G in exon 7 respectively.

Three patients $\left(\mathrm{P}_{10}, \mathrm{P}_{11}\right.$, and $\left.\mathrm{P}_{12}\right)$ did not carry any variation in the exon or exon-intron boundaries of PPT1 gene but their common clinical presentations like epilepsy, cerebral atrophy, and cerebellar atrophy indicated NCL. Their biochemical analysis depicting four times decrease in the PPT1 enzyme activity confirmed the NCL1 diagnosis. Since Sanger sequencing was uninformative, these patients were analyzed through MLPA. However, no large deletion/duplication was discovered in the PPT1 gene. In such cases, the possibility of deep intronic variations cannot be ruled out.

\section{Bi-directional sanger sequencing (TPP1 gene)}

As confirmed by biochemical investigation, 22 patients affected with NCL2 were analyzed for pathogenic variants in TPP1 gene. Overall, 15 variants, comprising seven missense variants, four frameshift variants, three nonsense variants, and one intronic variant were identified amongst 19 patients (Table 3).

\section{Known mutations detected in TPP1 gene}

The TPP1 gene analysis detected seven known mutations distributed amongst 11 patients. Patient $\mathrm{P}_{13}$ was identified with a heterozygous copy of the known mutation p.Met345Leu in exon 8 [23]. However, the second variant responsible for the disease was unidentified may 


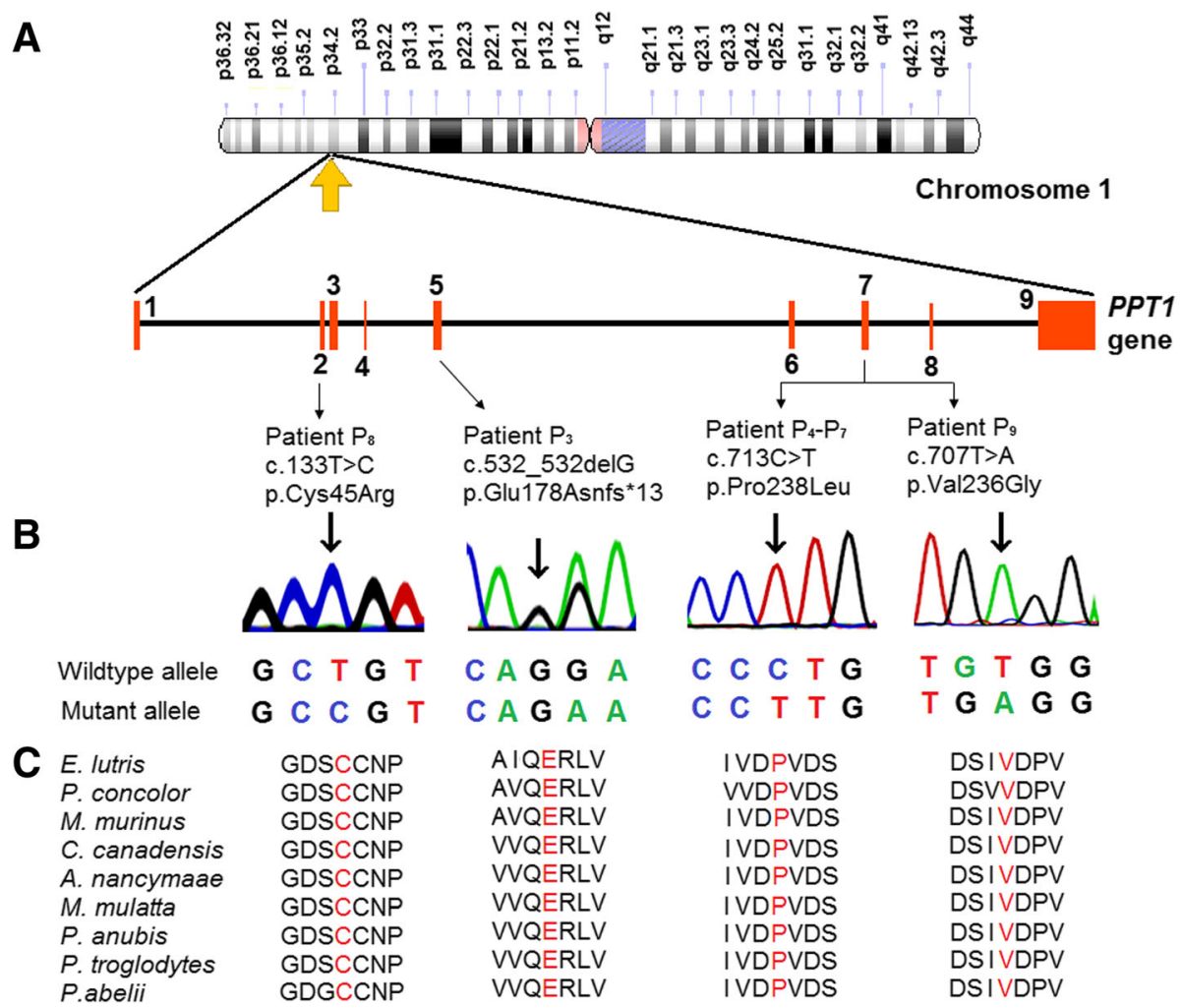

Fig. 1 Identification of novel variants in PPT1 gene. a Illustrative representation of the distributions of the novel variants identified in Indian NCL1 patients investigated in this study. $\mathbf{b}$ Sanger sequencing discovered one missense variant (p.Cys45Arg) in exon 2, two missense variants (p.Pro238Leu and p.Val236Gly) in exon 7, and one frameshift variation (p.Glu178Asnfs*13) in exon 5 of PPT1 gene. The common variant p.Pro238Leu was identified in 44\% of the patients. The point of variation is indicated by an arrow. c The multiple alignment of the protein sequence surrounding the novel variants against various orthologous sequence revealed the conservative status of the wildtype residues (marked red).

be due to its presence in the deep intronic region or due to possible large deletion/duplication. The patient has reduced TPP1 enzyme activity and clinical phenotypes like epilepsy, regression of mental and motor milestone, choreoathetosis, cerebral atrophy, and cerebellar atrophy were in line with the diagnosis. Patient $\mathrm{P}_{14}$ and $\mathrm{P}_{15}$ carried the homozygous missense mutation p.Arg339Trp and p.Arg339Gln respectively in exon 8 [2, 24]. Patient $\mathrm{P}_{16}$ was detected with a nonsense mutation p.Phe516Ter in exon 12 [25]. Patient $\mathrm{P}_{17}$ to $\mathrm{P}_{21}$ harbor a homozygous missense mutation p.Arg206Cys in exon 6 [26]. Patient $\mathrm{P}_{22}$ suffered NCL2 due to a homozygous nonsense mutation p.Arg208Ter in exon 6 [27].

\section{Novel variants detected in TPP1 gene}

Sanger sequencing revealed eight novel variants amongst nine patients (patients $\mathrm{P}_{23}$ to $\mathrm{P}_{31}$ ). This includes four frameshift variants, two missense variants, one intronic insertion, and one nonsense variant (Fig. 2). Patient $\mathrm{P}_{23}$ was found with a compound heterozygous variants p.Asn286Ser (known pathogenic) and p.Ser62Argfs"19 in exon 7 and exon
3 respectively [28]. In case of the patient $P_{24}$, a homozygous missense variant p.Arg152Ser was detected in exon 5. In the patient $\mathrm{P}_{25}$ and $\mathrm{P}_{26}$, the variant p.Tyr459Ser in exon 11 resulted in NCL2. A frameshift termination p.Ser153Profs*19 in exon 5 was identified in the patient $\mathrm{P}_{27}$. Sanger sequencing detected the patient $\mathrm{P}_{28}$ with a homozygous nonsense termination p.Tyr157Ter in exon 5. Patient $\mathrm{P}_{29}$ was compound heterozygous for the variants p.F230Sfs*28 in and p.Ile484Aspfs*7 in were detected in exon 7 and exon 12 respectively. An intronic variant g.2023_2024insT intron 4 was identified in the Patient $\mathrm{P}_{30}$ and $\mathrm{P}_{31}$.

However, no variation in the exonic or exon-intronic boundaries of TPP1 gene was detected in three patients $\left(\mathrm{P}_{32}, \mathrm{P}_{33}\right.$, and $\left.\mathrm{P}_{34}\right)$. These patients presented common clinical indications like epilepsy, cerebral atrophy, cerebellar atrophy, and visual impairment as indicated in NCL patients. Their biochemical analysis resulting in a significant decrease in the TPP1 enzyme activity confirmed the NCL2 diagnosis. Despite the uninformative Sanger sequencing results, these patients could not be 


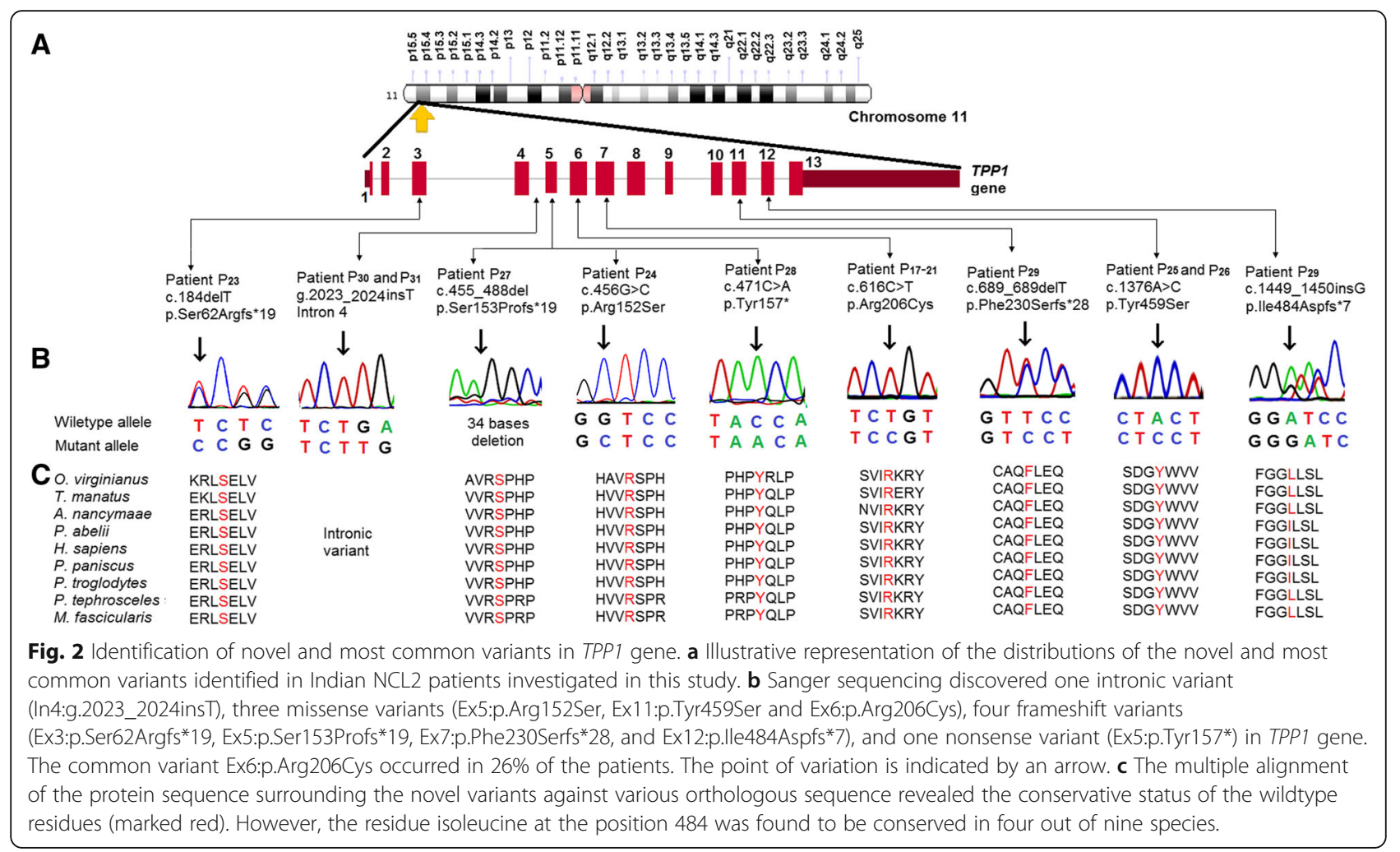

analyzed through MLPA due to unavailability of enough samples. In such cases, the possibility of deep intronic variations cannot be rule out.

\section{In silico analysis of the novel variants}

The in silico tools described above established the functional effects of the variants identified [see Additional file 2]. The novel variants were found to be disease causing. These predicting tools suggest the probably damaging and deleterious effect of the novel variants on protein function. These variants were found neither in the 1000 Genomes database nor in the Exome Aggregation Consortium (ExAc). The protein sequence alignment of Homo sapiens along with other species using Clastal Omega-an online multiple sequence alignment program suggests that these variations occurred at highly evolutionarily conserved and functionally active residual domain in the protein (Figs. 1 and 2).

The protein homology modeling of the missense point variants in the PPT1 gene (p.Cys45Arg, p.Val236Glu, and p.Pro238Leu) and TPP1 gene (p.Arg152Ser and p.Tyr459Ser) suggest their damaging effect at highly conserved residues. The variant p.Cys45Arg is in close proximity to Met41 and could affect the active site of PPT1 either by decreasing the oxyanion stabilization, altering the binding pocket or disrupting the active site by perturbing the position of Met41. The variant p.Val236Glu and p.Pro238Leu are very close to catalytic site
Asp233. This could affect the N-linked glycosylation process and cause conformational changes in the protein (Fig. 3).

The amino acid substitution in the variant p.Arg152Ser causes disruption in $\beta$-strand conformation, which might disturb processing of TPP1. While the variant p.Tyr459Ser probably compromise the active center and destabilizes hydrophobic pocket (Fig. 4).

The novel variants of PPT1 and TPP1 genes were screened in 100 control individuals, however, none carried the given variants. The most common mutation p.Pro238Leu observed in $44 \%$ of patients with NCL1 and p.Arg206Cys observed in $26 \%$ of patients with NCL2 were screened in 100 unrelated healthy subjects and were found to have normal allele. The novel variants identified in the present study are submitted to the NCBI ClinVar repository [see Additional file 3].

\section{Discussion}

Data presented here is the first study from India demonstrating the mutation spectrum of Batten disease (NCL1 and NCL2) in a large cohort. The given study reveals 34 cases of NCLs (12 with NCL1, and 22 with NCL2) with maximum NCL2 cases. Similarly, in a study by Santorelli et al., the highest numbers of cases confirmed the NCL2 (24\%) [29].

The patients' clinical appearance like seizures, myoclonic jerk, visual impairment, and neuroimaging 

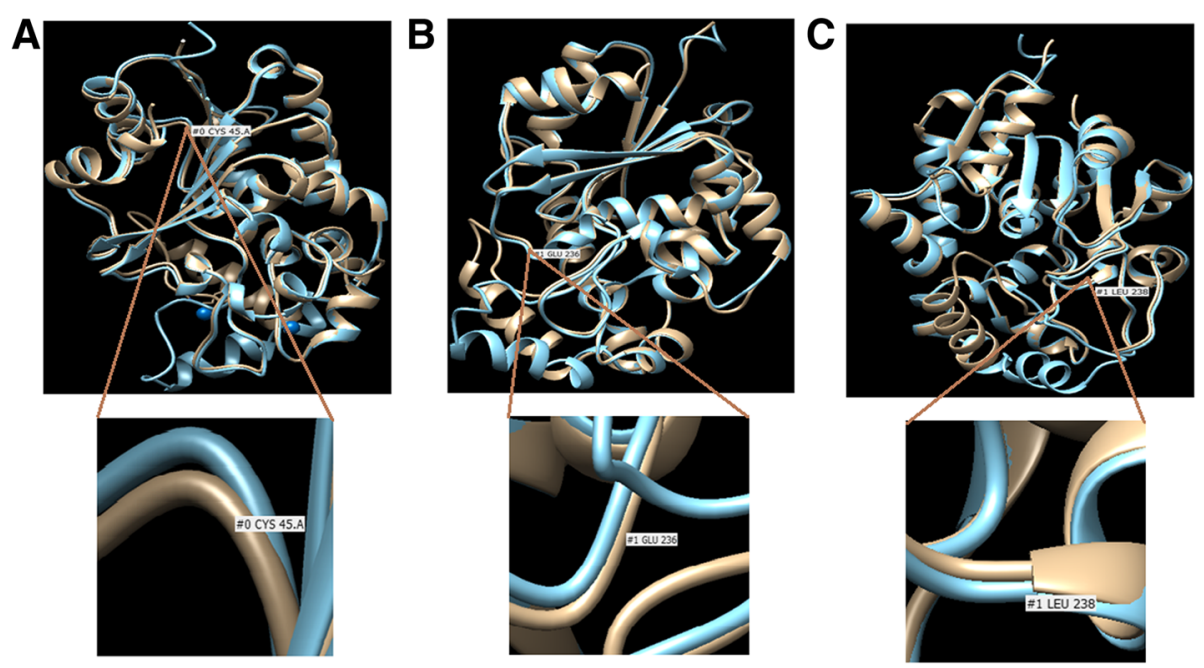

Fig. 3 Homology modeling of novel missense variants identified in PPT1 gene. The native structure (blue) and mutant structure (brown) are superimposed. a The model of p.Cys45Arg depicting the amino acid change from polar to basic at the codon number 45 (TGT-CGT). b The model of p.Val236Gly depicting the amino acid change from non-polar to acidic at the codon number 236 (GTG-GAG). c The model of p.Pro238Leu depicting the amino acide change from non-polar to hydrophobic at the codon number 238 (CCT-CTT). All the models reveal the conformational changes in the PPT1 protein structure.
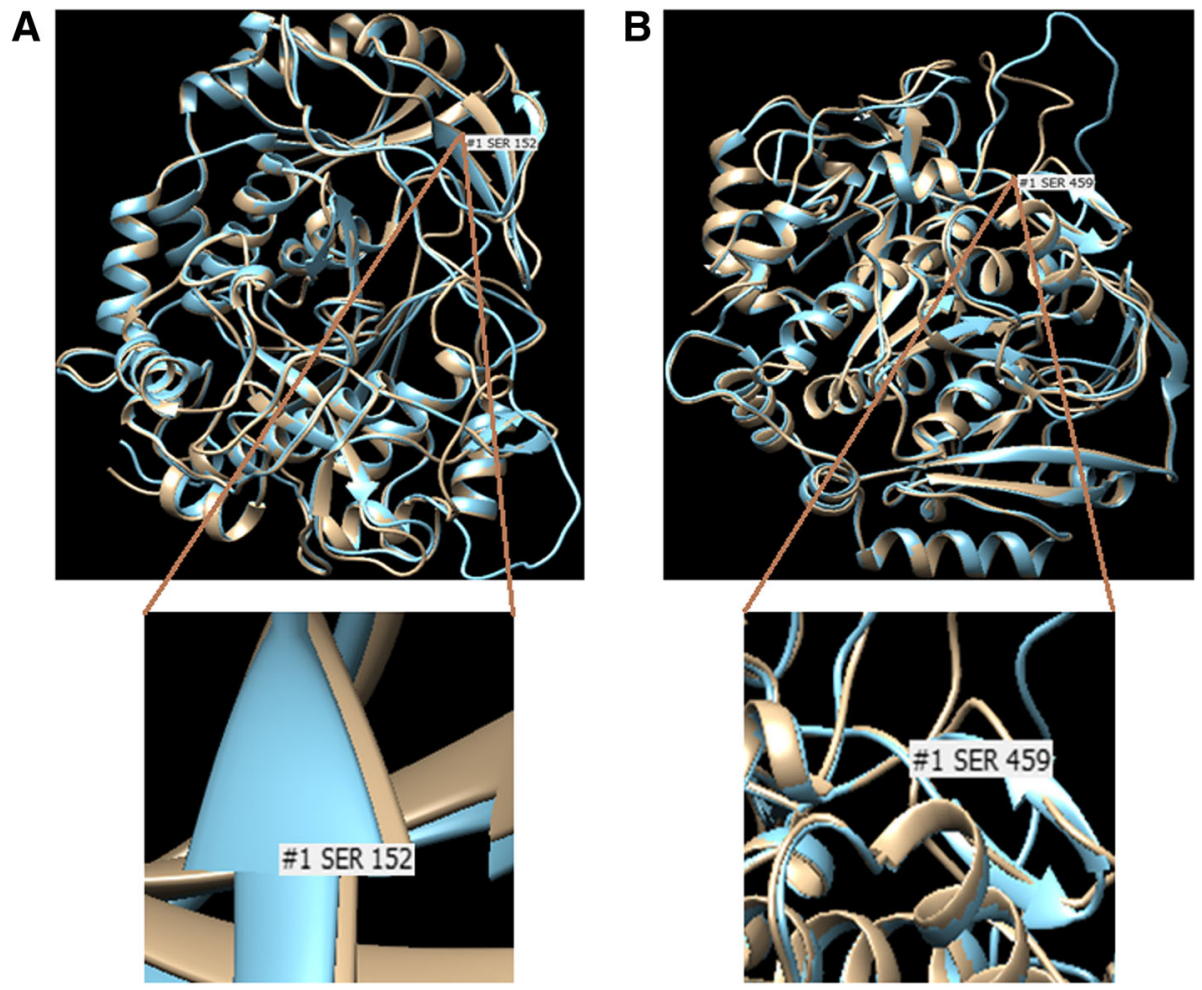

Fig. 4 Homology modeling of novel missense variants identified in TPP1 gene. The native structure (blue) and mutant structure (brown) are superimposed. a The model of p.Arg152Ser depicting the amino acid change from basic to polar at the codon number 152 (AGG-AGC). $\mathbf{b}$ The model of p.Tyr459Ser depicting the amino acid change from cyclic to non-cyclic at the codon number 459 (TAC-TCC). Both the models reveal the conformational changes in the TPP1 protein structure. 
examination showing cerebral atrophy and cerebellar atrophy were in concordance with the previously established phenotypes in NCL patients [3]. The mutations identified in this study resulted in a broader spectrum of clinical presentation and hence hampered the genotype-phenotype correlation in NCL patients. Such clinical presentations due to PPT1 and TPP1 gene mutations are also observed in the orthologous species. For instance, a study by Sanders et al. identified a homozygous mutation c.736_737insC in exon 8 of PPT1 gene in a canine presenting NCL-like signs including, visual impairment, disorientation, behavioral changes, lack of PPT1 activity in the brain, and accumulation of autofluorescent lysosomal inclusions with the granular osmiophilic deposit in neurons [30]. Also, a study by Mahmood et al. established that a homozygous TPP1 gene mutation in a zebrafish results in the progressive early onset of neurodegenerative phenotypes, small retina, accumulation of subunit c of mitochondrial ATP-synthase, and localized apoptotic cells death in the retina, optic tectum, and cerebellum [31].

Several country-specific mutations are reported in NCL1 and NCL2. The most common NCL1 mutation identified in Finland is p.Arg122Trp in PPT1 gene, which accounts for $98 \%$ Finish variants [12]. A study by Das et al. revealed two common mutations, p.Arg151Ter and p.Thr75Pro, in PPT1 gene of American NCL1 patients [32]. The absence of these common mutations in the present study of Indian patients suggests the molecular heterogeneity of NCL1 in India. In this study, a novel variant c.713C $>\mathrm{T}$ (p.Pro238Leu) was identified in the PPT1 gene of four unrelated NCL1 positive families (44\%) from the southern part of India. This suggests its possible founder effect in the Indian origin settlers. However, a detailed study in larger cohorts is essential.

In case of NCL2, two common mutations p.Arg208Ter and c.509-1G > C (as per old nomenclature T523-1G > C) accounting for approximately $60 \%$ of all identified TPP1 mutant alleles worldwide and at least one of these mutation can be identified in more than $75 \%$ of patients $[33,34]$. In the present study, one patient with NCL2 was identified with a homozygous p.Arg208Ter mutation but the variant c.509-1G $>\mathrm{C}$ was not observed in our cohort which indicate its uncommon occurrence in Indian NCL2 patients. The NCL2 country-specific mutation includes p.Gly284Val in Canada and p.Asp276Val in Argentina $[14,35]$. In the present study, a known pathogenic mutation p.Arg206Cys was observed most commonly in the unrelated NCL2 patients (26\%) suggesting its possible founder effect.

However, in the given study, the genetic diagnosis of about $17 \%$ of patients remained ambiguous. A similar percentage was also observed in previously published data were around $10 \%$ of patients were without any genetic identification [29]. This suggests that the deep intronic variants, large deletion or duplication in the NCL genes might also play a role in disease occurrence. In addition, as suggested by Santorelli et al., studying large informative families might identify new NCL genes and help in understanding NCLs molecular pathology [29].

A study by Das et al. established that a reduction in PPT1 and TPP1 enzyme activity ranges from 0 to $2.5 \%$ [33]. In the given study also, the reduction in these enzymes activity was from 0 to $2.8 \%$. Based on these biochemical observations, the therapeutic approaches are tested to regain the enzyme activity. For instance, a study in a canine model with TPP1-deficiency revealed that the administration of a recombinant adeno-associated virus (rAAV) expressing canine TPP1 in the ependyma resulted in elevation of TPP1 expression leading to delay in clinical presentation and extension of life span [36]. In addition, studies to diminish the clinical phenotypes of NCL have been directed. Tracy et al. reported an alternative approach of using stem cell based delivery of therapeutic components to the retina, as the systemic administration would be ineffective [37]. This study reported the inhibition of the retinal degeneration in the canine model after a single intravitreal administration of autologous bone marrow-derived stem cells transduced with a TPP1 expression construct [37].

\section{Conclusions}

The given study contributes four novel variants in $P P T 1$ gene and eight novel variants in TPP1 gene mutation spectrum. Our results with remarkable heterogeneity provide new insight into the molecular pathology of NCL1 and NCL2. In addition, it was observed that the novel variant p.Pro238Leu was common in Indian NCL1 patients while a known pathogenic mutation p.Arg206Cys was commonly observed in Indian NCL2 patients. This can give a new insight into the molecular pathology of NCL patients with Indian origin.

\section{Additional files}

Additional file 1: List of primers used for PPT1 and TPP1 gene sequencing. The exons and the exon-intron boundaries of both the genes were bidirectionally sequenced using the given set of primers. (DOCX $13 \mathrm{~kb}$ )

Additional file 2: In silico analysis of the functional effect of the variants identified in the patients with NCL1 and NCL2. The in silico tools predicting the effect of DNA variants, coding non-synonymous variants, amino acid substitution, and non-coding variants were employed to predict the functional effect of the variants identified in the given study. (DOCX $17 \mathrm{~kb}$ )

Additional file 3: ClinVar Accession ID of the novel variants generated in the given study. The variants identified through Sanger sequencing are reported in NCBI ClinVar database. The file provides accession ID and the links to an individual variant. (DOCX $13 \mathrm{~kb}$ ) 


\section{Abbreviations}

EXAc: Exome aggregation consortium; MLPA: Multiplex ligation-dependent probe amplification; NCBI: National Center for Biotechnology Information; NCL: Neuronal ceroid lipofuscinoses; PCR: Polymerase chain reaction; PPT1: Palmitoyl-protein thioesterase 1; TPP1: Tripeptidyl peptidase 1

\section{Acknowledgments}

We show our gratitude to the proband and their families for their support and without whose consent this study would not have been possible. We also appreciate the suggestions from Task Force members (Dr. Ratna Puri, Dr. Seema Kapoor, Dr. Ashwin Dalal, Dr. Subha Phadake, Dr. Girisha Katta, Dr. V Shankar) for referring patients at our institute. We also acknowledge Department of Health Research, Indian Council of Medical Research (ICMR), Government of India as a part of Multicentric National Task Force on Lysosomal storage disorders (Project No: GIA/31(ii)/2014-DHR) for supporting this work.

\section{Funding}

This work is supported by Department of Health Research, Indian Council of Medical Research (ICMR), Government of India, as a part of Multicentric National Task Force on Lysosomal storage disorders (Project No: GIA/31(ii)/ 2014-DHR). The funding agency was not involved in the study design, specimen collection, analysis, interpretation and preparation of the manuscript.

\section{Availability of data and materials}

The dataset generated and/or analyzed during the current study is available in the ClinVar repository [see Additional file 3].

\section{Authors' contributions}

Conceived and designed the experiments: JS, FS. Clinical analysis: JS, MK (Mahesh Kamate), NG, MK (Madhulika Kabra), SM, SN, AT, VJ, RS. Laboratory workup and data analysis: MM, RB, DP. Manuscript writing: DP, MM. Critical revisions and approval of final manuscript: JS, FS. All authors reviewed and approved the final manuscript.

\section{Ethics approval and consent to participate}

- The present study has been approved by the institutional ethics committee [FRIGE's Institute of Human Genetics] with approval number FRIGE/IEC/14/2016 dated 19th November 2016. This process is in accordance with the declaration of Helsinki.

- As per the institutional ethics committee guidelines, a written informed consent for investigation and publication of the data was obtained from the parents/guardian of the patients.

- An informed consent for publication was also obtained from the individuals included in the submission [This was in accordance with the requirement of the institutional ethics committee].

\section{Consent for publication}

A written informed consent was obtained from parents/guardian for publication of the patients' clinical details and/or clinical images. A copy of the written consent is available for review by the editor of this journal.

\section{Competing interests}

The authors declare that they have no competing interests.

\section{Publisher's Note}

Springer Nature remains neutral with regard to jurisdictional claims in published maps and institutional affiliations.

\footnotetext{
Author details

'FRIGE's Institute of Human Genetics, FRIGE House, Jodhpur Gam Road, Satellite, Ahmedabad, Gujarat 380015, India. ${ }^{2}$ Department of Pediatric Neurology, KLES Prabhakar Kore Hospital, Belgaum, Karnataka 590010, India. ${ }^{3}$ Division of Genetics (Pediatrics), All India Institute of Medical Sciences, New Delhi 110029, India. ${ }^{4}$ Usha-Deep Children Neurology and Epilepsy clinic, Ahmedabad 380014, India. ${ }^{5}$ Department of Pediatric Genetics, Amrita Institute of Medical Science and Research Centre, Kochi, Kerala 682041, India. ${ }^{6}$ Department of Neurology, Lokmanya Tilak Medical College, Sion Hospital, Mumbai, Maharashtra 400022, India. ${ }^{7}$ Department of Neurology, Santokba
}

Durlabhji Hospital, Jaipur 302015, Rajasthan, India. ${ }^{8}$ Ankur Neonatal Nursery, Ahmedabad 380009, Gujarat, India.

\section{Received: 14 August 2018 Accepted: 27 November 2018 \\ Published online: 12 December 2018}

\section{References}

1. Jalanko A, Thomas B. Neuronal ceroid lipofuscinosis. Biochim Biophys Acta. 2009;1793(4):697-709.

2. Kohan R, Carabelos MN, Xin W, Sims K, Guelbert N, Cismondi IA, et al. Neuronal ceroid lipofuscinosis type CLN2: a new rationale for the construction of phenotypic subgroups based on a survey of 25 cases in South America. Gene. 2013;516(1):114-21.

3. Mole SE, Cotman SL. Genetics of the Neuronal Ceroid Lipofuscinoses (Batten disease). Biochim Biophys Acta. 2015;1852(10 PtB):2237-41.

4. Kohlschütter A, Williams RE, Goebel HH, Mole SE, Boustany R-M, van Diggelen OP, et al. NCL Diagnosis and Algorithms. In: Mole SE, Williams RE, Goebel HH, editors. The Neuronal Ceroid Lipofuscinoses (Batten Disease). 2nd edition. Oxford: Oxford University Press; 2011. P.24-34.

5. Williams RE, Mole SE. New nomenclature and classification scheme for the neuronal ceroid lipofuscinoses. Neurology. 2012;79(2):183-91.

6. Bennett MJ, Rakheja D. The neuronal ceroid-lipofuscinoses. Dev Disabil Res Rev. 2013;17(3):254-9.

7. Noskova L, Stránecky V, Hartmannová H, Pristoupilova A, Baresova V, Ivanek $R$, et al. Mutations in DNAJC5, encoding cysteine-string protein alpha, cause autosomal dominant adult-onset neuronal ceroid lipofuscinosis. Am J Hum Genet. 2011;89(2):241-52.

8. Vantaggiato C, Redaelli F, Falcone S, Perrotta C, Tonelli A, Bondioni S, et al. A novel CLN8 mutation in late-infantile-onset neuronal ceroid lipofuscinosis (LINCL) reveals aspects of CLN8 neurobiological function. Hum Mutat. 2009; 30(7):1104-16.

9. NCL Mutation Database. http://www.ucl.ac.uk/ncl. Assessed 28 July 2018.

10. Glykys J, Sims, KB. The Neuronal Ceroid Lipofuscinosis Disorders. In: Swaiman KF, Ashwal S, Ferriero DM, Schor NF, Finkel RS, Gropman AL, Pearl PL, Shevell Ml, editors. Swaiman's Pediatric Neurology (Sixth Edition) Elsevier; 2017. p.390-404.

11. Lyly A, Marjavaara SK, Kyttälä A, Uusi-Rauva K, Luiro K, Kopra O, et al. Deficiency of the INCL protein Ppt1 results in changes in ectopic F1-ATP synthase and altered cholesterol metabolism. Hum Mol Genet. 2008;17(10): 1406-17.

12. Vesa J, Hellsten E, Verkruyse LA, Camp LA, Rapola J, Santavuori P, et al. Mutations in the palmitoyl protein thioesterase gene causing infantile neuronal ceroid lipofuscinosis. Nature. 1995;376(6541):584-7.

13. Hellsten E, Vesa J, Olkkonen VM, Jalanko A, Peltonen L. Human palmitoyl protein thioesterase: evidence for lysosomal targeting of the enzyme and disturbed cellular routing in infantile neuronal ceroid lipofuscinosis. EMBO J. 1996;15(19):5240-5.

14. Warrier V, Vieira M, Mole SE. Genetic basis and phenotypic correlations of the neuronal ceroid lipofusinoses. Biochim Biophys Acta. 2013;1832(11): 1827-30.

15. van Diggelen OP, Keulemans JL, Winchester B, Hofman IL, Vanhanen SL, Santavuori $\mathrm{P}$, et al. A rapid fluorogenic palmitoyl-protein thioesterase assay: pre- and postnatal diagnosis of INCL. Mol Genet Metab. 1999;66(4):240-4.

16. Sohar I, Sleat DE, Jadot M, Lobel P. Biochemical characterization of a lysosomal protease deficient in classical late infantile neuronal ceroid lipofuscinosis (LINCL) and development of an enzyme based assay for diagnosis and exclusion of LINCL in human specimens and animal models. J. Neurochem. 1999;73(2):700-11.

17. Miller SA, Dykes DD, Polesky HA. A simple salting out procedure for extracting DNA from human nucleated cells. Nucleic Acids Res. 1988; 16(3):1215.

18. Sheth J, Pancholi D, Mistri M, Nath P, Ankleshwaria C, Bhavsar R, et al. Biochemical and molecular characterization of adult patients with type I Gaucher disease and carrier frequency analysis of Leu444Pro - a common Gaucher disease mutation in India. BMC Med Genet. 2018;19(1):178.

19. Sheth J, Bhavsar R, Gandhi A, Sheth F, Pancholi D. A case of Raine syndrome presenting with facial dysmorphy and review of literature. BMC Med Genet. 2018;19(1):76.

20. Waliany S, Das AK, Gaben A, Wisniewski KE, Hofmann SL. Identification of three novel mutations of the palmitoyl-protein thioesterase-1 (PPT1) 
gene in children with neuronal ceroid-lipofuscinosis. Hum Mutat. 2000; 15(2):206-7.

21. NCBI ClinVar database. https://www.ncbi.nlm.nih.gov/clinvar/variation/ 196249/. Accessed 24 July 2018.

22. Kousi M, Lehesjoki AE, Mole SE. Update of the mutation spectrum and clinical correlations of over 360 mutations in eight genes that underlie the neuronal ceroid lipofuscinoses. Hum Mutat. 2012;33(1):42-63.

23. NCBI ClinVar database. https://www.ncbi.nIm.nih.gov/clinvar/variation/ 305509/. Accessed 24 Jul 2018.

24. Ohba C, Osaka H, lai M, Yamashita S, Suzuki Y, Aida N, et al. Diagnostic utility of whole exome sequencing in patients showing cerebellar and/or vermis atrophy in childhood. Neurogenetics. 2013;14(3-4):225-32.

25. ExAC browser beta. http://exac.broadinstitute.org/variant/11-6636099-CAA-C. Accessed 24 July 2018.

26. Berry-Kravis E, Sleat DE, Sohar I, Meyer P, Donnelly R, Lobel P. Prenatal testing for late infantile neuronal ceroid lipofuscinosis. Ann Neurol. 2000; 47(2):254-7.

27. Barisić N, Logan P, Pikija S, Skarpa D, Blau N. R208X mutation in CLN2 gene associated with reduced cerebrospinal fluid pterins in a girl with classic late infantile neuronal ceroid lipofuscinosis. Croat Med J. 2003;44(4):489-93.

28. Tsiakas K, Steinfeld R, Storch S, Ezaki J, Lukacs Z, Kominami E, et al. Mutation of the glycosylated asparagine residue 286 in human CLN2 protein results in loss of enzymatic activity. Glycobiology. 2004;14(4):1C-5C.

29. Santorelli FM, Garavaglia B, Cardona F, Nardocci N, Bernardina BD, Sartori S, et al. Molecular epidemiology of childhood neuronal ceroid-lipofuscinosis in Italy. Orphanet J Rare Dis. 2013:8:19.

30. Sanders DN, Farias FH, Johnson GS, Chiang V, Cook JR, O'Brien DP, et al. A mutation in canine PPT1 causes early onset neuronal ceroid lipofuscinosis in a Dachshund. Mol Genet Metab. 2010;100(4):349-356.

31. Mahmood F, Fu S, Cooke J, Wilson SW, Cooper JD, Russell C. A zebrafish model of CLN2 disease is deficient in tripeptidyl peptidase 1 and displays progressive neurodegeneration accompanied by a reduction in proliferation. Brain. 2013:136(Pt 5):1488-507.

32. Das AK, Becerra CH, Yi W, Lu JY, Siakotos AN, Wisniewski KE, et al. Molecular genetics of palmitoyl-protein thioesterase deficiency in the U.S. J Clin Invest. 1998;102(2):361-70.

33. Zhong N. Neuronal ceroid lipofuscinoses and possible pathogenic mechanism. Mol Genet Metab. 2000;71(1-2):195-206.

34. Bodzioch M, Aslanidis C, Kacinski M, Zhong N, Wisniewski KE, Schmitz G. Rapid detection of the two most common CLN2 mutations causing classical late infantile neuronal ceroid lipofuscinosis. Clin Chem. 2000;46(10):1696-9.

35. Kohan R, Pesaola F, Guelbert N, Pons P, Oller-Ramírez AM, Rautenberg G, et al. The neuronal ceroid lipofuscinoses program: $A$ translational research experience in Argentina. Biochim Biophys Acta. 2015;1852(10 Pt B):2301-11.

36. Katz ML, Tecedor L, Chen Y, Williamson BG, Lysenko E, Wininger FA, et al. AAV gene transfer delays disease onset in a TPP1-deficient canine model of the late infantile form of Batten disease. Sci Transl Med. 2015;7(313): 313 ra180.

37. Tracy CJ, Whiting RE, Pearce JW, Williamson BG, Vansteenkiste DP, Gillespie $L E$, et al. Intravitreal implantation of TPP1-transduced stem cells delays retinal degeneration in canine CLN2 neuronal ceroid lipofuscinosis. Exp Eye Res. 2016;152:77-87.

Ready to submit your research? Choose BMC and benefit from:

- fast, convenient online submission

- thorough peer review by experienced researchers in your field

- rapid publication on acceptance

- support for research data, including large and complex data types

- gold Open Access which fosters wider collaboration and increased citations

- maximum visibility for your research: over $100 \mathrm{M}$ website views per year

At $\mathrm{BMC}$, research is always in progress.

Learn more biomedcentral.com/submissions 